\title{
Vigilance Estimation by Using Electrooculographic Features
}

\author{
Jia-Xin Ma, Li-Chen Shi and Bao-Liang Lu Senior Member, IEEE
}

\begin{abstract}
This study aims at using electrooculographic (EOG) features, mainly slow eye movements (SEM), to estimate the human vigilance changes during a monotonous task. In particular, SEMs are first automatically detected by a method based on discrete wavelet transform, then linear dynamic system is used to find the trajectory of vigilance changes according to the SEM proportion. The performance of this system is evaluated by the correlation coefficients between the final outputs and the local error rates of the subjects. The result suggests that SEMs perform better than rapid eye movements (REM) and blinks in estimating the vigilance. Using SEM alone, the correlation can achieve 0.75 for off-line, while combined with a feature from blinks it reaches 0.79 .
\end{abstract}

\section{INTRODUCTION}

Nowadays we are confronting a considerable amount of accidents by the loss of vigilance. For many kinds of jobs, especially for vehicle drivers, aeroplane pilots and crew, security forces and high accuracy process controllers, losing vigilance may cause serious accidents. In this case, it is important to develop a method for continuously monitoring people's states in real time, thus to prevent the occurrence of drowsiness, sleepiness or fatigue.

The research of sleep has a relatively long history, and drowsiness also belongs to a part of it. In 1968, Rechtschaffen and Kales proposed rules to classify the sleep stages according to the features extracted from electroencephalogram (EEG), electrooculogram (EOG), and electromyogram (EMG). They divide sleep into five stages (S1, S2, S3, S4, and REM), plus the state of wakefulness (W). Drowsiness occurs during the transition between $\mathrm{W}$ and S1. Estimating the vigilance loss due to drowsiness is a similar process as classifying the sleep stages. To solve this problem, EEG power spectral density, and EOG features like rapid eye movements (REM), slow eye movements (SEM), blinks, and eyelid movements are widely used in many studies [1]-[6].

While there are different measurements for the validity of the methods in these studies, it is a direct and objective way to calculate the correlation coefficient between the selected features and the subject's local error rate during a monotonous task. Local error rate is defined as the current probability that the subject will fail to respond to a presented

This work was partially supported by the National Natural Science Foundation of China (Grant No. 60773090 and Grant No. 90820018), the National Basic Research Program of China (Grant No. 2009CB320901), the National High-Tech Research Program of China (Grant No. 2008AA02Z310 and Grant No. 2008AA02Z315), and the Science and Technology Commission of Shanghai Municipality (Grant No. 09511502400)

J. X. Ma, L. C. Shi and B. L. Lu are with the Center for Brain-Like Computing and Machine Intelligence, Department of Computer Science and Engineering, Shanghai Jiao Tong University and MOE-Microsoft Key Laboratory for Intelligent Computing and Intelligent Systems, Shanghai Jiao Tong University, 800 Dong Chuan Road, Shanghai 200240, China target within a time window with a constant width [7]. Recently, a method based on fractal dimension theory has high correlation between EEG and local error rate [4]. Another example uses video equipment to detect blinks and then calculates the correlation with local error rate [5].

In this research, we mainly evaluate our work by the correlation between the vigilance and the SEMs. SEMs and EEG have been found tightly related as indices of vigilance [8], [9]. Although EEG is a relatively fast and direct functional reflection and is the most promising item in the sleep related studies, disadvantageously, it is easily corrupted by noises originated by other simultaneously ongoing processes like muscle movements. Since EOG signal is much more intense, we can utilize it without de-artefact procedure which is usually time-consuming. In addition, EOG needs much fewer non-contactless electrodes (for detecting SEMs, two electrodes are enough), and those electrodes would not disturbed by hair on the scalp, which means lower requirements for the signal gathering and amplifying device. Besides SEMs, we also check the validity of some other features, like REMs and blinks.

This paper consists of two parts. The first is SEM detection based on the method proposed in [10], using discrete wavelet transformation. The second part is smoothing by linear dynamic system. All the processes can be done in real time.

\section{MATERIALS AND METHODS}

\section{A. Experiment description}

Seven sessions of EEG and EOG recordings from 4 men and 1 woman aged around 20 were extracted using the NeuroScan system.

Each subject underwent a lack of sleep during the last night and was in a fatigue state, which was in order to actually get the transition curve from wake to a sleepy state. A total of 62 EEG channels and 2 EOG channels were recorded from electrodes placed according to the extended 10-20 system with a reference on the top of the scalp. The signals were recorded in 32-bit resolution, sampled at $500 \mathrm{~Hz}$, and filtered between 0.1 and $100 \mathrm{~Hz}$.

The subjects sit in a comfortable chair, about 2 feet away from an LCD screen in a room without noise. The screen continuously displayed traffic signs of random colors, which included red, yellow, blue and green, by the NeuroScan Stim ${ }^{2}$ software. There were more than 40 kinds of traffic signs in each color. The sign was displayed for $0.5 s$, then the screen turned black for a $5 \sim 7 s$ interval before the next sign was presented. Each subject had a pad with four buttons in his or her hands. When a sign appeared, the subject was supposed to push the button of the same color with the sign. At the 
same time, the system recorded subject's reaction time and correctness of each pushing. The whole procedure lasted for more than 1 hour.

In our research, the EOG signal (vertical and horizontal) were used for data analysis. The local error rate series calculated by a 2-minute time window were used as the vigilance index (with a step of $8.096 \mathrm{~s}$, which is consistent with EOG features).

\section{B. SEM detection algorithm}

The SEM detection algorithm originally proposed by Magosso et al. [10] is made up of three steps. (1) discrete wavelet decomposition of EOG signals; (2) computation of energy from wavelet coefficients; (3) a discriminant function and a threshold used to calculate the final output. The authors have used the algorithm in 24-h EOG recordings [11]. In our application, we implement it feasible for on-line usage, thus we can detect SEMs in real time. The details of the method are as follows.

1) Wavelet decomposition: In the first step, discrete wavelet transformation (DWT) is used to decompose the input signal into components of different frequency bands. The input signal is the difference between the right horizontal EOG and the left horizontal EOG. We first down-sample the $500 \mathrm{~Hz}$ original signal to $125 \mathrm{~Hz}$. Then the signal is processed by a 10-level DWT by Daubechies order 4 (db4) wavelet. This process divides the signal into 10 components of different scales that represent different frequency ranges of the original EOG. Scale 1 principally encompasses frequencies in the range $31.25 \sim 62.5 \mathrm{~Hz}$. Higher scales are centered at half the frequency of the previous scale. While SEMs are typically $0.2 \sim 0.6 \mathrm{~Hz}$, scale $7 \sim 10$ are necessary to capture the band of SEMs.

2) Energy computation: Wavelet coefficients can be used to compute energy associated with each components. The general idea of the method is to compute the energy of scale $7 \sim 10$, to divide it by the total energy of the signal, and to get the energy ratio of the SEM band. If the result is higher than a threshold, the corresponding time interval is considered as an SEM period.

In order to compute the energy, we introduce the wavelet energy function as follows,

$$
E_{i}=\sum_{j=1}^{M / 2^{i}}\left|d_{i j}\right|^{2} \text { for } i=1,2, \ldots, 10
$$

where $E_{i}$ is the total energy of the component of $i$ th scale, and $d_{i j}$ is the wavelet coefficient of $i$ th scale and $j$ th instance. Generally, for an input discrete signal with a length $M$, the number of wavelet coefficient in each scale is approximately equal to $M / 2^{i}$. When we compute the energy in a fixed time interval, it is necessary to unify the coefficient resolution of each scale. Here we use scale 6 as the standard. For $i<6$, the coefficients are closer in time so they need to be grouped, and for $i>6$, the coefficients are sparse in the time axis so they need to be scattered.
3) The discriminant function: After we get the energy of each scale, there is a discriminant function judging whether the energy ratio is larger than a threshold,

$$
f=\frac{\sum_{i=7}^{10} W_{i} E_{i}}{\sum_{i=3}^{5} W_{i} E_{i}+\sum_{i=7}^{10} W_{i} E_{i}},
$$

where $W_{i}$ is the weight of scale $i$. In this formula scale 1 , 2 , and 6 are discarded because scale 1 and 2 include high frequency noises, and scale 6 is ambiguous in its behavior. The weights in this function and the threshold were trained through a number of subjects in the original method, by comparing with visually inspection and validation. In this paper, we directly adopt the trained weights and the threshold in [10].

In order to process the signal on-line, we execute the DWT on a fixed length of time with a fixed step, instead of executing it on the whole signal. The length is $128 \times 32$ points, which is $32.384 s$, and the step is $128 \times 8$ points, which is $8.096 \mathrm{~s}$. Since in scale 10 the number of coefficients is supposed to be $M / 1024,32.384 s$ just contain 4 wavelet coefficients and $8.096 \mathrm{~s}$ contain one, so we use these time lengths. Also note that for short signal section, the number of coefficients is not approximately equal to $M / 2^{i}$ but larger than this because the coefficients are calculated by convolution. Therefore, we only keep $M / 2^{i}$ coefficients in the middle, and discard the other.

The output of this algorithm is the SEM proportion within each time window, 8.096s in this case, ranged from 0 to 1.

\section{Dynamic model for smoothing}

Since normally used moving average is not suitable for on-line processing, we introduce the linear dynamical system (LDS) for off-line and on-line vigilance estimation to process the SEM proportion curve and to replace the original smooth procedure. Its details can be found in our another work [12].

Assume that the input signal consists of two parts, which are vigilance-related and vigilance-unrelated. LDS can reduce the influence of vigilance-unrelated part. It finds out the vigilance trajectory from the index space without any label information. Compared with static methods, LDS can utilize the time dependency of vigilance changes. Table I lists the correlation coefficients between error rates and SEMs, which processed by off-line LDS, on-line LDS, and moving average, respectively.

\section{TABLE I}

PERFORMANCE COMPARISON OF LDS AND MOVING AVERAGE

\begin{tabular}{|c|c|c|c|}
\hline Session & Off-line LDS & On-line LDS & Moving average \\
\hline 1 & 0.72 & 0.68 & 0.65 \\
\hline 2 & 0.59 & 0.54 & 0.61 \\
\hline 3 & 0.92 & 0.84 & 0.86 \\
\hline 4 & 0.74 & 0.66 & 0.56 \\
\hline 5 & 0.85 & 0.76 & 0.77 \\
\hline 6 & 0.66 & 0.56 & 0.48 \\
\hline Average & 0.75 & 0.67 & 0.65 \\
\hline
\end{tabular}



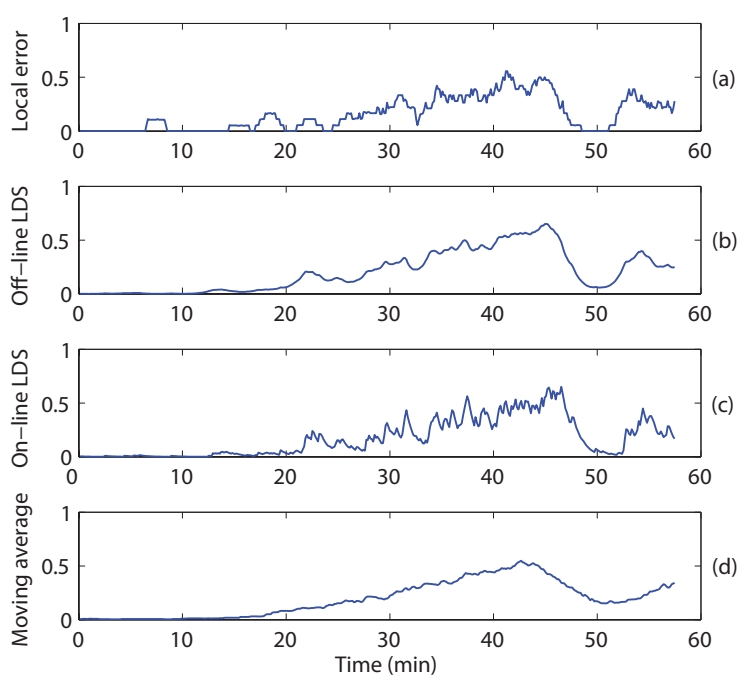

Fig. 1. Comparison of local error rate and SEMs with different smoothing methods, Session 3. Here, (a) is the local error rate; (b) is SEMs + off-line LDS; (c) is SEMs + on-line LDS; and (d) is SEMs + 2 min moving average. Each output is properly re-scaled to a range of $0 \sim 1$, the same below.

Fig. 1 illustrates the output of the algorithm. From the figure we can see that the shape and the trend of (b) is similar to (a), where the peaks and the valleys coincide very well in these two figures. For on-line LDS the average correlation is 7 percent lower than off-line LDS, but from the figure we can see it reflects more details than off-line smoothing, and its shape is not changed. For the moving average method, although the correlation is a little higher than on-line LDS, its actual result in this figure seems only worse since it reflects few details. Overall, on-line LDS performs better than moving average.

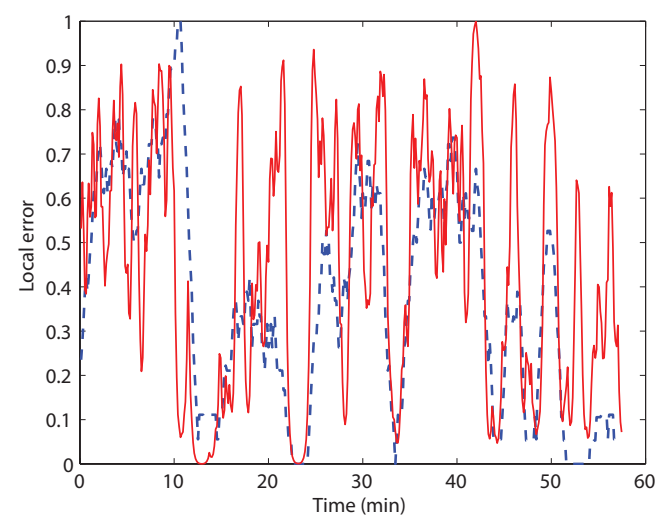

Fig. 2. Comparison of local error rate (blue dash) and SEMs + off-line LDS (red solid), Session 2.

Fig. 2 is about session 2, where blue dash line is for local error rate and red solid line is for off-line LDS. Its correlation is only 0.59 , the lowest among all the subjects. However, we can see that the peaks and valleys of the two curves also coincide well. The low correlation is mainly due to the difference of the amplitude, and the sudden drop of SEM curve when the local error rate reaches 1 , which we will discuss later.

\section{Feature combination}

Many studies use more than one feature for vigilance estimation and get better results than using single feature. Therefore, we also try to add additional features in our research.

Blinks have been mentioned to be used to detect fatigues, and six different features of blink are validated in [3], which are blink duration, blink interval, positive and negative peaks, closing time, and reopening time. The paper stated that blink duration, positive and negative peaks, and reopening time are useful for the fatigue detection. Also, various models are used to combine blink duration and blink frequency [5]. Moreover, REMs are also reported as related to vigilance. In [13], the author found the relationship between REMs and EEG during the sleep onset.

In this research, we extract REMs and blink features from EOG, then use off-line LDS to process those features, and calculate the correlation coefficients. For REMs, we adopt the automatic detection method in [13]; for blinks, we use a simple threshold method. Results show that they does not work as well as SEMs.
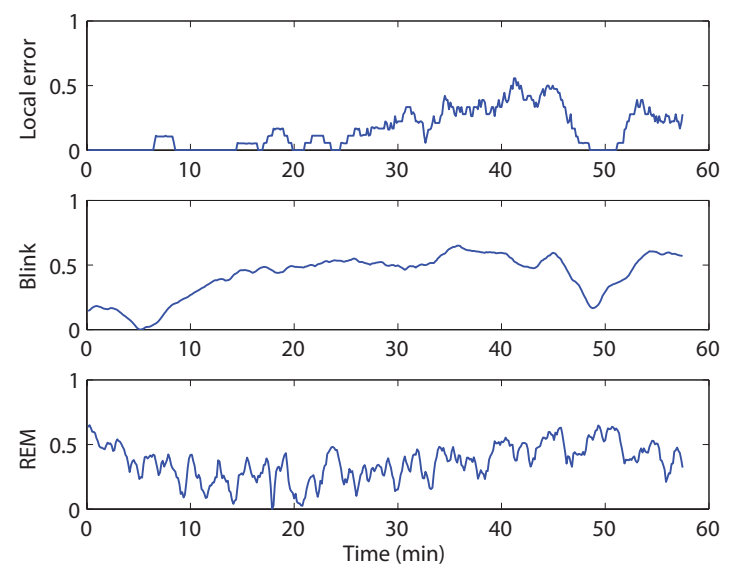

Fig. 3. Comparison of local error rate, negative peak (blink), and REMs, Session 3.

From Fig. 3, we can see that REMs seem not able to match the trend and shape of the error curve. For blinks, we find that negative peak is a usable feature for most of the sessions, but it only reflect the general trend but not enough details of the error rate curve. This means REMs and single feature of blinks are not so reliable as SEMs.

Next, we linearly combine the negative peaks and SEMs. For those subjects to whom the negative peak does not work, we replace it by another feature. The results are shown in Table II.

\section{DISCUSSION AND CONCLUSION}

Table I and Figs. 1 and 2 show that we can get satisfactory results by using single SEM feature. Although the average 
TABLE II

PERFormANCE COMPARISON OF SINGLE AND COMBINED

FEATURES

\begin{tabular}{|c|c|c|c|}
\hline Session & SEMs & Negative peaks & Linear combination \\
\hline 1 & 0.72 & 0.76 & 0.84 \\
\hline 2 & 0.59 & $0.49^{\mathrm{a}}$ & 0.73 \\
\hline 3 & 0.92 & 0.65 & 0.92 \\
\hline 4 & 0.74 & 0.60 & 0.70 \\
\hline 5 & 0.85 & $0.76^{\mathrm{b}}$ & 0.86 \\
\hline 6 & 0.66 & 0.48 & 0.69 \\
\hline Average & 0.75 & 0.62 & 0.79 \\
\hline
\end{tabular}

a Blink duration.

${ }^{\mathrm{b}}$ Reopening time.

correlation value seems not so high, which is 0.75 for offline LDS, SEMs match the error rate of subjects very well. Adding another feature makes the correlation increase to 0.79. Since we have not adjusted the parameters of the SEM detection algorithm, we believe that if we have trained the weights and threshold on our samples, the result will be better.

Among all the subjects in our experiments, only one is excluded from our results, since that subject has a very low correlation. The reason is highly probably due to that he has fallen asleep during the experiment, whereas others have not. However, it does not mean that SEMs do not work properly in this case. From Fig. 4, we can see that there are two periods where his error rate reaches 1 , which means he is in a state of sleep. Correspondingly, the SEM curve has a sudden drop, to a level of near zero. This phenomenon may indicate that the subject has entered the S2 sleep, because in this stage SEMs are greatly decreased. From this figure we can conclude that SEMs can work as an error rate indicator only before the subject enter S2 sleep. But it does not matter since we only consider the $\mathrm{W}$ and $\mathrm{S} 1$ stages in the vigilance related research.

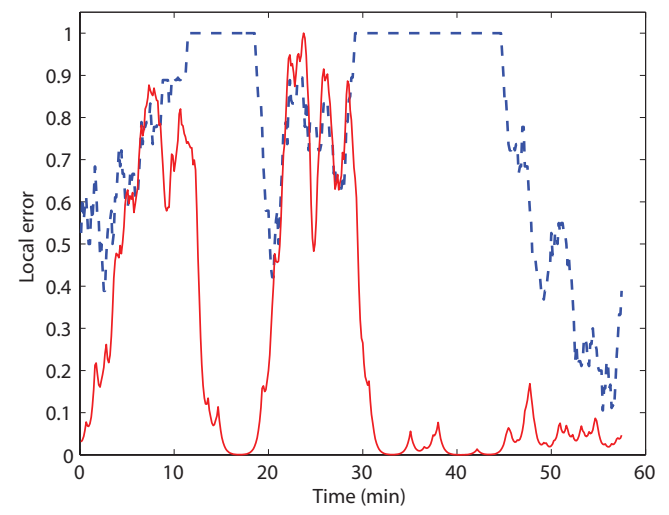

Fig. 4. Comparison of local error rate (blue dash) and SEMs with off-line LDS (red solid), the subject who fell asleep.

An EEG experiment is also executed on these subjects in [12], in which principal component analysis is used on EEG and some predominant components are combined to get the result. Compared with EEG, the EOG correlation is lower and has a larger variance. This result is predictable since we believe that EEG reflects the most direct information of people's state and is more reliable especially in an undisturbed experiment environment. In addition, the time window and step used in EEG feature can be shorter than that used in EOG. In our research we use a 8.096s step and many EOG related studies use $10 s$ since a short step for EOG will cause the result lack of statistical significance. On the contrary, in the EEG test, the step can be $2 s$. Which means faster response to the vigilance changes of subjects.

However, EOG, especially SEM feature is still useful in vigilance estimation because of the reasons mentioned in Part I. The experiment results also show that SEMs can best match the error rate curve among all the EOG features tested. In our following studies we will test more subjects and try to build a robust model. Also we would like to combine EEG and EOG features for the application on vigilance estimation.

\section{REFERENCES}

[1] M. Golz, D. Sommer, M. Holzbrecher, and T. Schnupp, "Detection and Prediction of Driver's Microsleep Events", 14th International Conference of Road Safety on Four Continents. Retrieved: February, $2,2009$.

[2] M. Atienza, J.L. Cantero, R. Stickgold, and J.A. Hobson, "Eyelid movements measured by Nightcap predict slow eye movements during quiet wakefulness in humans", Journal of Sleep Research, vol. 13, pp. 25-29, 2004.

[3] Y.S. Kim, H.J. Baek, J.S. Kim, H.B. Lee, J.M. Choi, and K.S. Park, "Helmet-based physiological signal monitoring system", European Journal of Applied Physiology, vol. 105, pp. 365-372, 2009.

[4] S.P. Arjunan, D.K Kumar, and T.P. Jung, "Changes in Decibel Scale Wavelength Properties of EEG with Alertness Levels While Performing Sustained Attention Tasks", Proceedings of 31st International Conference of the IEEE Engineering in Medicine and Biology Society, Minneapolis, USA, pp. 6288-6291, 2009.

[5] K.F.V. Orden, T.P. Jung, and S.Makeig, "Combined eye activity measures accurately estimate changes in sustained visual task performance", Biological Psychology, vol. 52, pp. 221-240, 2000

[6] L.C. Shi, H. Yu, and B.L. Lu, "Dynamic Clustering for Vigilance Analysis Based on EEG", Proceedings of 30th International Conference of the IEEE Engineering in Medicine and Biology Society, Vancouver, BC Canada, pp. 54-57, 2008

[7] S. Makeig and M. Inlow, "Lapse in alertness: coherence of fluctuations in performance and EEG spectrum", Electroencephalography and Clinical Neurophysiology, vol. 86, pp. 23-35, 1993.

[8] L. D. Gennaro, M. Ferrara, F. Ferlazzo, and M. Bertini, "Slow eye movements and EEG power spectra during wake-sleep transition", Clinical Neurophysiology, vol. 111, pp. 2107-2115, 2000.

[9] E. Magosso, M. Ursino, F. Provini, and P. Montagna, "Wavelet Analysis of Electroencephalographic and Electro-Oculographic Changes During the Sleep Onset Period", Proceedings of 29th International Conference of the IEEE Engineering in Medicine and Biology Society, Lyon, France, pp. 4006-4010, 2007.

[10] E. Magosso, F. Provini, P. Montagna, and M. Ursino, "A wavelet based method for automatic detection of slow eye movements: A pilot study", Medical Engineering \& Physics, vol. 28, pp. 860-875, 2006.

[11] E. Magosso, M. Ursino, A. Zaniboni, F. Provini, and P. Montagna, "Visual and computer-based detection of slow eye movements in overnight and 24-h EOG recordings", Clinical Neurophysiology, vol. 118 , pp. 1122-1133, 2007

[12] L.C. Shi and B.L. Lu, "Off-Line and On-Line Vigilance Estimation Based on Linear Dynamical System and Manifold Learning", To appear in the Proceedings of 32nd International Conference of the IEEE Engineering in Medicine and Biology Society, Buenos Aires, Argentina

[13] K. Hyoki, M. Shigeta, N. Tsunob, Y. Kawamuro, and T. Kinoshita, "Quantitative electro-oculography and electroencephalography as indices of alertness", Electroencephalography and clinical Neurophysiology, vol. 106, pp. 213-219, 1998. 